\title{
Blasenkatheter gezielt einsetzen: seltener, kürzer, sicherer!
}

\author{
Stephanie Züllig \\ Dr. sc. nat., Programmleitung progress! Sicherheit bei Blasenkathetern, Patientensicherheit Schweiz
}

Transurethrale Dauerkatheter, kurz Blasenkatheter, sind im medizinischen Alltag allgegenwärtig. Obwohl wir wissen, dass Blasenkatheter eine Eintrittspforte für Bakterien darstellen und das Risiko für eine Bakteriurie pro Tag um 3-7\% erhöhen, erhalten 20-25\% aller hospitalisierten Patientinnen und Patienten einen Blasenkatheter. Rund ein Viertel davon erleidet eine Harnwegsinfektion, woraus sich in 3-5\% der Fälle eine Sepsis entwickelt. Etwa die Hälfte der 350000 jährlich in der Schweiz gelegten Blasenkatheter haben keine klare medizinische Indikation.

Diese Zahlen sind eindrücklich, insbesondere wenn man bedenkt, dass die Einlage des Katheters oftmals mit Komplikationen, wie beispielsweise mechanische Traumata der Harnröhre, verbunden ist. Katheterassoziierte nosokomiale Infektionen oder Verletzungen bleiben nicht ohne Konsequenzen: Sie bedürfen einer Folgebehandlung, verlängern den Spitalaufenthalt und erhöhen die Kosten. Internationale Studien zeigen, dass mit spezifischen Interventionen sowohl die Häufigkeit als auch die Dauer der Kathetereinsätze massgeblich gesenkt werden können. Damit einhergehend reduziert sich auch das Infektions- und Verletzungsrisiko.

In der Schweiz hat die Stiftung für Patientensicherheit die Thematik aufgenommen und zusammen mit Swiss-

Indikationen

\begin{tabular}{|c|c|}
\hline \multirow[t]{2}{*}{ Harnverhalt } & - akuter Harnverhalt \\
\hline & $\begin{array}{l}\text { - symptomatische chronische Ausflussobstruktion } \\
\text { PLUS >300 ml Restharn }\end{array}$ \\
\hline Urinmonitoring / Bilanzierung & $\begin{array}{l}\text { - im regelmässigen kurzen Intervall PLUS direkte } \\
\text { therapeutische Konsequenzen aus } \\
\text { der Bilanzierung }\end{array}$ \\
\hline \multirow[t]{4}{*}{ Operation } & - lange Operation (>4 h) \\
\hline & - perioperativ: aus operationstechnischen Gründen \\
\hline & - Urogenitalchirurgie und/oder Beckenbodenchirurgie \\
\hline & - epidurale/peridurale Anästhesie/Analgesie \\
\hline Dekubitalulzera PLUS Inkontinenz & $\begin{array}{l}\text { - Dekubitus Grad III oder IV oder Hauttransplantate } \\
\text { sacral/perineal PLUS Inkontinenz* }\end{array}$ \\
\hline Prolongierte Immobilisation & $\begin{array}{l}\text { - aus medizinischen Gründen*, insbesondere } \\
\text { zur Schmerzreduktion }\end{array}$ \\
\hline \multirow[t]{2}{*}{ Palliation PLUS Komfort } & $\begin{array}{l}\text { - terminal-palliative Situation PLUS Störung } \\
\text { der Blasenfunktion PLUS/ODER Störung der } \\
\text { normalen Miktion* }\end{array}$ \\
\hline & $\begin{array}{l}\text { - bei hohem Leidensdruck PLUS auf Wunsch des } \\
\text { informierten Patienten }\end{array}$ \\
\hline
\end{tabular}

* Nach Ausschöpfung von Alternativmethoden zur kontrollierten Urinableitung noso das nationale Pilotprogramm progress! Sicherheit bei Blasenkathetern initiiert. Dabei geht es um die Sensibilisierung von Fachpersonen und die Implementierung eines Interventionsbündels in Schweizer Spitälern zur Reduktion von Blasenkathetern und katheterassoziierten Komplikationen. Die Projektziele sind:

- Seltener: Kathetereinlage nur mit klarer Indikation

- Kürzer: Katheter sobald wie möglich wieder entfernen

- Sicherer: Katheter korrekt einlegen und pflegen

Um die Zielvorgaben zu erreichen, implementieren sieben Pilotspitäler ein Interventionsbündel, das aus den folgenden Elementen besteht:

1. Indikationsliste

Die Indikationsliste ist evidenzbasiert und führt Situationen auf, in denen ein Blasenkatheter gerechtfertigt ist (siehe Box). Eine Negativliste dient der Abgrenzung. Nach Möglichkeit kommen Alternativmethoden zum Einsatz.

2. Tägliche Überprüfung der Indikationsstellung Ein Reminder erinnert daran, die Indikation zu überprüfen. Ist sie nicht mehr gegeben, ist der Katheter am selben Tag zu entfernen.

3. Schulung des Personals

Ein Refresher erneuert das Wissen zur aseptischen Arbeitsweise und technisch einwandfreien Katheterisierung. Das Training bietet Gelegenheit zum Üben oder zum Überprüfen der Fähigkeiten.

Die Implementierung beginnt diesen Herbst und wird von einer Sensibilisierungskampagne begleitet. Ergebnisse aus den Evaluationserhebungen, wie die Anzahl Katheterisierungen oder die Häufigkeit von Komplikationen, erlauben das Verbesserungspotential abzuschätzen und die Wirksamkeit der Interventionen aufzuzeigen. Wie das Interventionsbündel am besten in den klinischen Alltag zu übernehmen ist, beschreibt die Handlungsempfehlung: Schriftenreihe Nr. 9, Sicherheit bei Blasenkathetern. Die Handlungsempfehlung kann über www.patientensicherheit.ch bezogen werden.

\section{Literatur}

Die Literatur zu diesem Artikel findet sich online unter www.saez.ch $\rightarrow$ Aktuelle Ausgabe oder $\rightarrow$ Archiv $\rightarrow 46$. 


\section{Ausgewählte Referenzen:}

Magill SS, Edwards JR, Bamberg W, Beldavs ZG, Dumyati G, Kainer MA, et al. Multistate point-prevalence survey of health care-associated infections. N Engl J Med 2014 Mar 26;370(13):1198-208.

Saint S. Clinical and economic consequences of nosocomial catheter-related bacteriuria. Am J Infect Control 2000;28(1):68-75.

Gould CV, Umscheid CA, Agarwal RK, Kuntz G, Pegues DA, and the Healthcare Infection Control Practices Advisory Board (HICPAC). Guideline for Prevention of Catheter- Associated Urinary Tract Infections 2009. Infection Control and Hospital Epidemiology 2010 Apr 1;31(4):319-26.
Fakih MG, George C, Edson BS, Goeschel CA, Saint S. Implementing a national program to reduce catheter associated urinary tract infection: a quality improvement collaboration of state hospital associations, academic medical centers, professional societies, and governmental agencies. Infect Control Hosp Epidemiol 2013 Oct;34(10):1048-54.

Meddings J, Saint S, Fowler KE, Gaies E, Hickner A, Krein SL, et al. The Ann Arbor Criteria for Appropriate Urinary Catheter Use in Hospitalized Medical Patients: Results Obtained by Using the RAND/UCLA Appropriateness Method. Ann Intern Med 2015 May 5;162(9_Supplement):S1-S34. 